\title{
TEORÍA DE LA GRAMÁTICA. LA CONCEPCIÓN DE EUGENIO COSERIU
}

\author{
HEIDI ASCHENBERG \\ Universidad de Heidelberg
}

La gramática es un tema central en la obra de Coseriu al que no cesó de volver a lo largo de su vida sin elaborarlo, sin embargo, de forma exhaustiva y sistemática. Los numerosos ensayos que dedicó a la teoría de la gramática así como al estudio de cuestiones empíricas sobre todo con respecto a las lenguas románicas reflejan su preocupación constante por una teoría gramatical coherente y por su aplicación ${ }^{1}$. Se pueden considerar estos trabajos como esbozos, segmentos e ilustraciones de una teoría integral anclada profundamente en su pensamiento lingüístico. Al estudiar estos textos más de cerca el lector no se dará cuenta solamente de las múltiples conexiones que tiene esta parte con las demás partes del edificio lingüístico de Coseriu, sino que notará también que la gramática forma parte de los cimientos de este edificio.

Las contribuciones de Coseriu a la teoría de la gramática, al contrario que sus trabajos por ejemplo sobre la semántica estructural, no han recibido el interés que merecen. Esto se explica ciertamente por la falta de una monografía suya sobre este tema. Voy a tratar de esbozar en lo que sigue el perfil de su concepción de gramática y de su fundación teórica en su pensamiento lingüístico.

\section{GRAMÁTICA GENERAL VS. GRAMÁTICA DE UNA LENGUA CONCRETA}

El término de gramática según Coseriu tiene dos acepciones fundamentales: significa en primer lugar «técnica del hablar», es decir saber gramatical del hablante «correspondiente a una lengua determinada» que este mismo actualiza en la comunicación con otros individuos (Coseriu 1987², 130). Coseriu denomina a esta gramática «gramática objeto» (ib.) porque constituye el objeto del análisis lingüístico. La segunda acepción, complemento de la primera, se refiere a la «descripción o investigación» del saber gramatical; con respecto a la gramática del hablante es, como dice Coseriu, gramática como «metalenguaje» (ib.).

\footnotetext{
${ }^{1}$ Véase, por ejemplo, Coseriu 1976; 1977; 1987; 1981; 1984; 1986; 1987; 1990.
} 
La distinción entre gramática general y gramática de una lengua concreta, claro está, se sitúa al nivel de la segunda acepción puesto que delimita las perspectivas distintas que puede adoptar el lingüista en la investigación gramatical. Mientras que la gramática general es una disciplina teórica que desarrolla y define los conceptos necesarios para el análisis empírico, potencialmente aplicables a cualquier lengua, la gramática de una lengua concreta - como dice ya su denominación - tiene siempre que ver con una lengua determinada, o, en el caso de la gramática contrastiva, con dos lenguas (id. 1986, 105 s.). La gramática de una lengua concreta puede ostentar perfiles diversos: puede proceder sincrónica o diacrónicamente, puede ser normativa, destinada a finalidades didácticas o puede ser descriptiva, científica, analizando las técnicas gramaticales con categorías lingüísticas (ib., 108 s.). En Introducción a la lingüística (1986) Coseriu menciona «como forma especial de gramática descriptiva» la gramática estructural o funcional (ib., 109), que en otros trabajos constituye el centro de sus reflexiones sobre gramática.

\section{GRAMÁTICA Y SEMÁNTICA}

Para entender la concepción coseriana de la gramática es necesario exponer en primer lugar algunas nociones básicas de su teoría semántica, porque teoría gramatical y teoría semántica están estrechamente entrelazadas en su obra. Se aprecia ya aquí que los diversos dominios temáticos de su pensamiento lingüístico no pueden considerarse aislados, sino que forman un todo fundado en su teoría general del hablar humano. Por esto Coseriu vuelve siempre en sus diversos trabajos a las distinciones fundamentales de su teoría para localizar las cuestiones respectivas y precisar sus propias perspectivas. Estas distinciones funcionan como una red conceptual estructurando y ligando las diversas partes del conjunto.

Con respecto a los diversos tipos de lo semántico, Coseriu propone, como se sabe, una distinción tripartita que corresponde a la delimitación de tres niveles del lenguaje (hablar en general, lengua concreta, discurso o texto):

1. la designación o «la referencia a lo extralingüístico» (Coseriu 1987² 135), contenido lingüístico específico del hablar en general;

2. el significado, «contenido dado en y por una lengua como tal»;

3. el sentido, «plano semántico propio y exclusivo del 'texto'»(ib., $135 \mathrm{~s}$.) $)^{2}$.

Mientras que la gramática general o universal se basa con respecto a lo semántico en la designación (ib., 136) la gramática funcional en cuanto gramática de una lengua concreta tiene que ver con el significado. Es, así pues, este tipo de contenido semántico lo que tenemos que analizar más en detalle.

Se puede decir que Coseriu propone una visión bastante amplia de lo semántico propio de la lengua concreta. Lo que entiende por significado no abarca solamente contenidos disponibles y delimitados en el léxico de una lengua, sino también diversos tipos de contenidos específicamente gramaticales, sea de valor morfemático, sea de valor oracional.

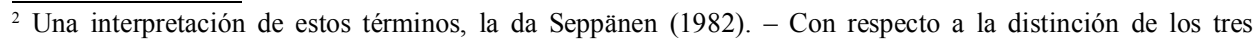
niveles del lenguaje véase Coseriu 1981, 269-286; la distinción de los tres tipos de contenido semántico en correspondencia con los tres niveles del lenguaje id. $1994^{3}, 58-67$.
}

Odisea, $\mathrm{n}^{\circ} 3,2003$ 
El primer tipo de significado y, claro está, el más evidente es el significado léxico, «que corresponde al qué de la aprehensión del mundo extralingüístico» (ib., 136). Coseriu ilustra este tipo de sentido con palabras de la misma raíz, pero pertenecientes a diferentes categorías verbales como: caliente - calor - calentar.

El significado léxico, proporcionado por la raíz cal- es el mismo en estas palabras. El segundo tipo de significado, el significado categorial, corresponde «al cómo de la aprehensión del mundo extralingüístico». Se puede otra vez recurrir a las palabras ya citadas para explicarlo. La pertenencia de estas palabras a diversas categorías verbales refleja las diferencias en la percepción del mismo contenido designado y se pone de manifiesto en la formación diferente de los lexemas respectivos ${ }^{3}$.

El tercer tipo, el significado instrumental, define el significado de los morfemas, independientemente de si se trata de morfemas libres o ligados. Así, por ejemplo, la $-s$ en casa-s tiene el «significado 'plural'», o el artículo determinado en un sintagma como el hombre tiene el «significado 'actualizador'»(ib., 137).

El significado estructural se refiere al eje sintagmático del lenguaje, Coseriu lo denomina también «sentido sintáctico en sentido estricto». Este tipo de sentido resulta de la combinación de lexemas con morfemas dentro de la oración, como, por ejemplo, «singular», «plural», «activo», «pasivo» etc. (ib.).

El último tipo está ligado al nivel de la oración: es el sentido óntico; es decir «el valor existencial que se asigna al estado de cosas designado en una oración», por ejemplo «afirmativo», «negativo», «interrogativo», etc. (ib.).

Coseriu compara la relación entre significado estructural y óntico con la relación entre significado estructural y categorial, el primero correspondiente al qué, el segundo al cómo de lo aprehendido. Así en oraciones como Juan ha leído el libro. / ¿Ha leído Juan el libro? el significado estructural es idéntico; lo que es diferente en estas oraciones es el sentido óntico (ib., 138). A primera vista esta observación podría hacer pensar en una distinción análoga de la pragmática, la existente entre contenido proposicional e ilocución. Pero puesto que las distinciones de Coseriu no se refieren al acto de habla, al enunciado en una situación comunicativa concreta, sino a la oración como entidad del sistema, estas distinciones se refieren a niveles diferentes del lenguaje.

Coseriu ilustra los diversos tipos de sentido en un esquema muy útil (ib., 140):

\begin{tabular}{|l|l|}
\hline significado léxico & \multirow{3}{*}{ Léxico (y lexicología) } \\
\hline significado categorial & \\
\hline significado instrumental & \\
\hline significado estructural & \\
\hline significado óntico & \\
\hline
\end{tabular}

\footnotetext{
3 Coseriu considera las categorías verbales como categorías semánticas, potencialmente universales, puesto que representan solamente modos de aprehensión y no contenidos léxicos concretos de una lengua determinada. Véase el ensayo «Sobre las categorías verbales» publicado en Coseriu 1987², 50-79.
} 
Este esquema muestra la estructuración semántico-gramatical de las funciones lingüísticas y hace patentes a la vez los dominios respectivos de la lexicología y de la gramática.

\section{TEORÍA DE LA GRAMÁTICA}

No es fácil trazar las ideas esenciales de la concepción de gramática de Coseriu. Se trata de un modelo muy complejo basado en numerosas distinciones terminológicas, sin que éstas estén desarrolladas y localizadas coherentemente con referencia a un edificio teórico de la gramática. Se aprecia sin excepción en los ensayos dedicados a este tema un procedimiento extremamente analítico. Sin embargo se encuentra en diversos trabajos la idea de un sistema de la gramática, esbozado en algunas pocas líneas. Antes de entrar más en los detalles cabe anticipar algunas observaciones que ayudan a percibir más claramente la intención teórica del autor y el corte de su modelo.

Coseriu es un lingüista que en la elaboración de sus propias ideas se basa siempre en la tradición, «filtrándola», como dijo Christian Lehmann ${ }^{4}$. Con respecto a la teoría de la gramática destaca que se refiere sobre todo a autores que sostienen una interpretación semántica de las funciones gramaticales como Hervás, Humboldt, Steinthal, Misteli, Byrne, Winkler,

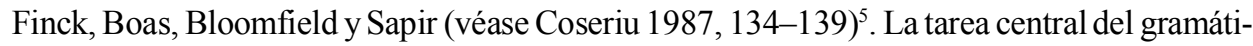
co según Coseriu consiste en el análisis de las estructuras de una lengua determinada al desprenderla de sus variedades. Por eso Coseriu denomina la gramática «lingüística de la homogeneidad», cuyo objeto tiene un carácter ideal (Coseriu 1990, 20 ss.).

En cuanto a los diversos tipos del significado (designación, significado, sentido) la gramática, tal como la concibe Coseriu, se ocupa exclusivamente del significado. Con esta orientación se distingue fundamentalmente de la gramática universal u onomasiológica que, como se mencionó ya más arriba, se basa en la designación estudiando las estructuras posibles del lenguaje en general (véase 1987, 133). Con respecto a su propio modelo, la gramática funcional, Coseriu rechaza la distinción entre sintaxis y gramática; para él las dos disciplinas forman un conjunto, no se puede separar la una de la otra (ib.). Considera la gramática funcional como un desarrollo en la tradición de la lingüística saussureana (véase Kabatek/ Murguía 1997, 158).

\subsection{El sistema de la gramática}

Hay en algunos trabajos de Coseriu unas pocas reflexiones sobre un sistema de gramática que, estructurado coherentemente, podría aplicarse a cualquier lengua ${ }^{6}$. Este sistema se compone de tres partes: la gramática constitucional, la funcional y la relacional.

La gramática constitucional es la gramática de la forma o morfología general que estudia la constitución material de las estructuras gramaticales (Coseriu 1987, 145) y eso en los diversos niveles del lenguaje. Estos niveles son los siguientes: elemento mínimo, pala-

\footnotetext{
${ }^{4}$ Lehmann 1988, 6.

${ }^{5}$ Destaca igualmente que la mayor parte de los autores mencionados trabajaron sobre todo en el dominio de la tipología del lenguaje.

${ }^{6}$ Véase, por ejemplo, Coseriu 1984, 42 ss.; 1987, 145 ss.; 1990, 43 ss.
}

Odisea, $n^{\circ} 3,2003$ 
bra, grupo de palabras, cláusula, oración, texto (Coseriu 1994³, 30 ss.). Son, como dice Coseriu, el resultado de una «generalización empírica» (ib., 30); puede ser que una lengua determinada no disponga de todos.

Así se puede discutir si el nivel de la palabra existe en latín. Para ilustrar el problema Coseriu compara la forma casas en español y en latín. En los dos casos se combinan elementos mínimos. Mientras que la $-s$ en la forma española casas marca solamente la pluralización del lexema, en la forma latina se expresa el acusativo del plural, es decir una función oracional, por ejemplo la de objeto o de indicación de la dirección. Lo mismo se nota en la forma de base casa que incluye también ya una función oracional, la de sujeto. Así pues las formas latinas no representan formas «puras», sino que están provistas siempre en sí mismas ya por una función oracional. Esto se muestra también en el nivel del grupo de palabras. En las lenguas románicas existe este nivel como nivel autónomo: l'homme pauvre / le pauvre homme en francés, son grupos de palabras, cuya constitución se da independientemente de su función en la oración. Esto no vale para los grupos de palabras en latín que, debido al carácter sintético de esta lengua, conllevan sin excepción una función oracional.

¿Qué entiende Coseriu por cláusula? Existe la cláusula como nivel autónomo de estructuración en una lengua si es posible integrar elementos en la oración que comentan a los otros. En la oración Probablemente lo hizo el adverbio comenta la proposición lo hizo al referirse a su validez (ib., 31 s.).

La relación lingüística fundamental correspondiente a la gramática constitucional es la relación combinatoria o sintagmática. Coseriu considera como tarea central de esta gramática el análisis de las relaciones sintagmáticas desde el nivel de los elementos mínimos hasta el nivel del texto.

La gramática funcional o sintaxis funcional representa según Coseriu la parte central de la gramática porque es el fundamento de las otras dos secciones, describiendo a la vez las estructuraciones materiales así como las relaciones entre estas mismas. En la gramática funcional llega a ser eficaz la idea según la cual la organización de los procedimientos semántico-gramaticales depende por principio del sistema de una lengua concreta. Se puede decir que la gramática funcional adopta una perspectiva semasiológica contrapuesta a la perspectiva onomasiológica de la gramática universal (véase Coseriu 1987, 140). La tarea principal de la gramática funcional según Coseriu consiste en averiguar los diversos significados gramaticales de una lengua dada y las relaciones opositivas de estos mismos refiriéndolos a unidades semántico-gramaticales más abstractas. Con tal fin la gramática funcional parte del postulado según el cual en una lengua a cada expresión le corresponde un significado unitario determinado (Coseriu 1987, 140). Coseriu ilustra esta idea con un ejemplo alemán: la preposición mit se puede emplear con referencia a sustantivos de carácter ontológico diferente: mit dem Messer (con el cuchillo), mit Ingrid (con Ingrid), mit Zucker (con azúcar), mit Vergnügen (con gusto); el significado unitario que forma la base de las diversas construcciones mit $x$ ( $\operatorname{con} x$ ), o, como dice Coseriu, «su identidad genérica» es «concomitancia» (ib., 141). La relación lingüística fundamental correspondiente a la gramática funcional es la relación paradigmática. Se trata de paradigmas de combinación o de paradigmas de sintagmas, puesto que una función gramatical se expresa siempre por medio de una combinación de elementos y no por elementos singulares. Así en la palabra casa-s el sustantivo con la marca del plural es portador del significado gramatical; lo mismo vale para

Odisea, $\mathrm{n}^{\circ} 3,2003$ 
el sintagma el hombre: no es el sustantivo el que por sí solo lleva el significado sino el sintagma completo (véase ib., 143). La gramática funcional tiene que investigar y comprobar los paradigmas que funcionan en los diversos estratos de la estructuración gramatical (paradigmas de la palabra, del grupo de palabras, de la oración) (véase Coseriu 1987, 132).

La gramática relacional está determinada por una orientación onomasiológica. Tiene que ver con problemas tratados también por la gramática transformacional. Su tarea es el análisis de las relaciones existentes entre los procedimientos de expresión que con medios semántico-gramaticales diferentes se refieren a un mismo contenido de pensamiento o a una mismaunidad dedesignación7. O, para traducir las palabras de Coseriu: la gramática relacional trata de las unidades de designación que se expresan por medio de signifiés diferentes funcionando como significados lingüísticos en paradigmas diferentes; así pues describe las relaciones entre estos paradigmas bajo el punto de vista de la designación (véase ib., 145) ${ }^{8}$. Coseriu menciona como ejemplos oraciones como $A$ es más alto que $B$ / $B$ es más pequeño que A. A se calló / A no dijo nada; A pega a $B$ / $B$ es pegado por $A$ (véase ib.).

Para ilustrar las diversas perspectivas de las tres secciones de la gramática Coseriu se refiere a las indicaciones locales del tipo Romae, Romam, Româ en latín y a sus equivalentes en las lenguas románicas (ib., 147):

\begin{tabular}{|l|l|l|l|}
\hline latín & español & francés & italiano \\
\hline Romae & en Roma & & \\
\cline { 1 - 2 } Romam & a Roma & à Rome & a Roma \\
\hline Romā & de Roma & de Rome & da Roma \\
\hline
\end{tabular}

Bajo el punto de vista de la gramática constitucional se aprecia en primer lugar la diferencia entre el latín y las lenguas románicas. Mientras que en latín las indicaciones locales se expresan a través de los casos, en las lenguas románicas se realizan a través de las preposiciones. En cuanto a la gramática funcional se puede comprobar que por un lado se corresponden el latín y el español y por el otro el francés y el italiano. Para analizar las diferencias relacionales Coseriu introduce expresiones sinónimas, pero de constitución diferente:

\begin{tabular}{|l|l|l|l|}
\hline latín & español & francés & italiano \\
\hline in urbe Romā & en la ciudad de Roma & dans la ville de Rome & nella città di Roma \\
\hline in urbem Romam & a la ciudad de Roma & à la ville de Rome & alla città di Roma \\
\hline ex urbe Romā & de la ciudad de Roma & de la ville de Rome & dalla città di Roma \\
\hline
\end{tabular}

\footnotetext{
${ }^{7}$ Esto vale, claro está, solamente bajo la condición de que las unidades de designación sean organizadas independientemente de las lenguas concretas. Véase con respecto a este tema Gauger 1982.

${ }^{8}$ El texto alemán se expresa en estos términos: „Die „relationelle» Grammatik schließlich befaßt sich mit den Bezeichnungseinheiten, die duch jeweils verschiedene Signifiés - d.h. die als Sprachbedeutungen in verschiedenen Paradigmen funktionieren - ausgedrückt werden, und demzufolge mit den Beziehungen zwischen diesen Paradigmen unter dem Gesichtspunkt der Bezeichnung» [...] (ib., 143).
}

Odisea, $n^{\circ} 3,2003$ 
Aquí, otra vez, se pone de manifiesto una diferencia entre el latín de una parte y las lenguas románicas de otra: en el paradigma de urbs Roma el latín recurre a construcciones de otro tipo empleando preposiciones. Las lenguas románicas sin embargo no cambian de construcción al seguir el mismo modelo.

Coseriu considera la gramática constitucional, la funcional y la relacional como secciones complementarias de la gramática. En cuanto al hablante representan diversos aspectos de su competencia (véase más abajo, 4.).

\subsection{Gramática transfrástica}

Coseriu ha esbozado sus ideas a propósito de la gramática transfrástica en su libro sobre lingüística textual (Coseriu 1994³). El objeto de la gramática transfrástica es el texto como nivel superior de la estructuración de la lengua (véase más arriba). Dicho de modo general, la gramática transfrástica analiza las reglas de una lengua determinada con respecto a la constitución del texto (ib., 29). Estas reglas se refieren a hechos que traspasan el límite de la oración (como, por ejemplo, el discurso directo, el discurso indirecto, la enumeración etc.) o que se refieren a fenómenos fuera de la oración (como los marcadores del discurso, la topicalización, diversos modos de la sustitución) (ib., 28).

En el esbozo de la lingüística transfrástica debemos apreciar también el esfuerzo del autor por encontrar categorías o principios que de modo coherente hagan patentes las estructuras de una lengua concreta. Estas categorías son la superordinación, la subordinación, la coordinación y la sustitución, que determinan relaciones existentes, sea entre los diversos niveles de la lengua (palabra, grupo de palabras, cláusula, oración, texto) sea dentro de un solo nivel. Por superordinación Coseriu entiende el hecho de que elementos de un nivel inferior puedan sustituir a unidades de un nivel superior. Así la forma llueve en español puede funcionar en cuanto palabra como oración, lo que no sería posible en el caso de al. regnet que requiere siempre el pronombre personal de la tercera persona es para formar una oración (es regnet). No se puede aplicar la relación de superordinación al nivel del texto puesto que este mismo representa el nivel superior de los niveles de la lengua.

La subordinación es la relación opuesta a la superordinación. En el caso de la subordinación una unidad de un nivel superior funciona como unidad de un nivel inferior. Grupos de palabras como, por ejemplo, con excepción de, por medio de, de acuerdo con se emplean como palabras y se pueden sustituir por las preposiciones excepto, por, según. En el nivel del texto Coseriu ilustra la subordinación por medio de la integración de un discurso en otro discurso como sucede por ejemplo en la cita, en el discurso directo y en el discurso indirecto. Cada lengua dispone de procedimientos específicos para estos casos. En alemán, por ejemplo, se emplea en la lengua escrita siempre el subjuntivo I (subjuntivo del presente) para marcar el discurso indirecto aún si tratamos de referirnos a un estado de cosas que ponemos en duda. En latín el discurso indirecto puede incorporarse en un texto como objeto directo. Por ejemplo: Caesar dixit: «Haec perfecta philosophia est quae [...] 》 puede transformarse en discurso indirecto del siguiente modo: Caesar hanc perfectam philosophiam (esse) iudicavit, quae [...] (véase ib., 224).

Por medio de la coordinación unidades del mismo nivel se unen para formar unidades más complejas. Cada lengua tiene procedimientos particulares para este tipo de relación, 
como se muestra ya en la combinación de elementos mínimos: el sintagma italiano con e senza libri no puede traducirse directamente al español, hay que cambiar la construcción: con libros y sin ellos (ib., 212).

El cuarto tipo, la sustitución, juega en general un papel central en las introducciones a la lingüística textual porque es ella la que contribuye en un alto grado a la cohesión o a la coherencia del texto y porque esto representa un ligamiento transfrástico importante. Coseriu considera la sustitución como un procedimiento anafórico o catafórico que funciona en todos los niveles, desde los elementos mínimos hasta los textos. Así podemos referirnos a un texto completo retomándolo por medio de las partículas no y sí. Un medio de sustitución económico y de uso muy frecuente es la pronominalización; otras posibilidades son los diversos modos de retomar un elemento léxicamente, por ejemplo de modo implícito: Llegamos a un pueblo. Estaba cerrada la iglesia (ib., 216). En este caso, iglesia remite implícitamente a pueblo, porque, como sabemos, hay iglesias en muchos pueblos.

Para Coseriu la gramática transfrástrica representa un enfoque importante de investigación lingüística, que sin embargo no corresponde a su propia concepción de la lingüística textual. La base de su teoría sobre lingüística textual es el texto en cuanto nivel del lenguaje en general, no en cuanto nivel de estructuración semántico-gramatical de una lengua determinada como en la gramática transfrástica. Basada en la hermeneútica, la lingüística textual de Coseriu analiza los diversos procedimientos expresivos que constituyen el sentido del texto, como los diversos tipos de relaciones semióticas y los diversos entornos a los cuales remiten los signos del texto.

\subsection{Gramática contrastiva}

Destaca en los trabajos de Coseriu sobre gramática la frecuente contraposición de ejemplos tomados del latín, de las lenguas románicas y del alemán. Como hemos visto, el análisis gramatical, según él, tiene que hacer patentes las estructuras semánticogramaticales de las lenguas concretas (véase más arriba, 2.). El procedimiento contrastivo tiene la ventaja de poner de relieve más nítidamente no sólo las categorías que le dirigen, sino también las estructuras respectivas de las expresiones y de las lenguas comparadas.

En su ensayo «Alcances y límites de la gramática contrastiva» Coseriu presenta más en detalle sus ideas sobre la comparación sincrónica de las lenguas y sus métodos. Vamos a estudiar en lo que sigue cómo está ligado este esbozo de la gramática contrastiva con su teoría general de gramática.

La gramática contrastiva tiene en primer lugar objetivos prácticos, está concebida para el aprendizaje de lenguas extranjeras. Como la teoría de la traducción, la gramática contrastiva supone un «tertium comparationis», un «contenido de pensamiento» según Coseriu, que hace posible la comparación $\left(1987^{2}, 81\right.$ s.). ¿Pero cómo se pueden identificar estos supuestos contenidos de pensamiento? ¿Y en qué medida dependen ellos mismos de la estructura de las lenguas concretas? Llegamos aquí a ‘uuno de los problemas más difíciles de la lingüística» (ib., 83). Coseriu propone la distinción entre contenidos de pensamiento no separables y contenidos de pensamiento separables 
de las lenguas concretas. Los primeros representan «configuraciones primarias del mundo», comparables al significado léxico; forman un estrato estrechamente unido con las lenguas. Los segundos corresponden «al hablar por medio de las lenguas sobre un mundo ya configurado lingüísticamente», comparables al significado oracional. Así, por ejemplo, las oraciones I like this, questo mi piace, esto me gusta expresan aproximadamente el mismo contenido de pensamiento, lo que no vale para to like, piacere y gustar (véase ib.).

La gramática contrastiva estudia en el habla los diversos procedimientos expresivos pertenecientes a dos lenguas diferentes que se refieren a situaciones análogas o a los mismos hechos. Asimismo, tiene que contar con el hecho de que las mismas «designaciones oracionales o los mismos significados de habla» pueden expresarse por medio de «categorías» diferentes entendidas como «significados»o «funciones» de lengua (véase ib., 86). Así, por ejemplo, a la diferenciación temporal en español entre pretérito imperfecto y pretérito indefinido tenía / tuvo corresponde en alemán una diferenciación léxica: ich hatte / ich bekam. Coseriu considera el procedimiento estructural o funcional como el más apropiado para la gramática contrastiva, porque describe por definición «el paso de los significados a las funciones lingüísticas propiamente dichas» (ib.). Propone una reforma de los planteamientos y métodos de la gramática contrastiva sobre una base estructuralista (véase ib., 95). $\mathrm{Y}$ es exactamente aquí donde podemos fijar el punto de contacto con su teoría gramatical. Para precisar su concepción se basa en los conceptos y en parte también en los ejemplos que expone en la teoría gramatical. Así retoma

1. la distinción de los estratos gramaticales (unidad mínima, palabra, grupo de palabras, cláusula, oración $)^{9}$;

2. la definición de las relaciones entre los estratos (superordinación, subordinación, coordinación y sustitución);

3. la idea de un sistema gramatical constituido por tres tipos de gramática complementarios (gramática constitucional, funcional, relacional);

4. los conceptos de la estructuración de la técnica idiomática (norma de lengua, sistema, tipo lingüistico) (véase ib., 97 s.) desarrollados en los escritos sobre lingüística general y aplicados en la teoría de la semántica estructural.

No es necesario volver a las tres primeras delimitaciones conceptuales, puesto que ya se han explicado en lo esencial. Pero debemos precisar lo que dice Coseriu con respecto a la estructuración de la técnica idiomática porque se localiza desde aquí más claramente el objeto de la gramática contrastiva. Como se sabe, la distinción entre norma y sistema de Coseriu representa una diferenciación del concepto saussureano de la langue. El término de norma no tiene un sentido prescriptivo, sino que representa el nivel más bajo en la jerarquía de las invariantes del hablar, abarcando el conjunto de las técnicas expresivas convencionalizadas en una comunidad lingüística. El sistema se sitúa a un nivel más abstracto, corresponde «a los procedimientos distintivos y a las funciones opositivas» subyacentes a una lengua (ib., 107). La noción de tipo lingüistico no se refiere a diversas lenguas, de modo que podría servir de base para algo como una clasificación de lenguas. Significa el nivel más alto de la estructuración de una sola

\footnotetext{
$\overline{9}$ Menciona el texto como estrato eventual dentro del campo de la gramática contrastiva.
} 
lengua. Contiene «las clases y categorías de procedimientos y funciones» de una lengua como dice Coseriu (ib., 107) ${ }^{10}$.

Las diferencias tipológicas entre las lenguas, aunque científicamente importantes, no lo son para la gramática contrastiva que tiene fines prácticos o didácticos. La diferencia entre norma y sistema de la lengua sin embargo es imprescindible para el aprendizaje de la lengua, porque tiene que ver con la aceptabilidad del hablar. Es posible que lo que es idiomáticamente correcto según los principios del sistema, no corresponda a las reglas de la norma, a lo que se dice normalmente en un determinado contexto lingüístico. Para ilustrar este punto de vista con respecto a la gramática contrastiva Coseriu da algunos ejemplos. Así, el equivalente del al. natürlich en italiano y español, en el plano del sistema, es naturalmente. Pero hay contextos en los que natürlich no se puede traducir por naturalmente porque esto no correspondería a la norma de los dos otras lenguas. En estos casos se emplea por ejemplo si capisce que, claro está que, por supuesto o desde luego (ib., 107 s.). Lo mismo vale para el al. aus Versehen, que, en el nivel del sistema, tiene como equivalentes en italiano per (una) svista y en español por (un) descuido. Pero estos equivalentes no se usan en cualquier contexto: hay situaciones en las que, conforme a la norma de su lengua, los italianos dicen senza volerlo y los españoles sin quererlo para reproducir el al. aus Versehen. Estos ejemplos demuestran la necesidad de distinguir entre sistema y norma también en la gramática contrastiva. Con respecto al hablante las nociones de sistema y norma acentúan dos aspectos diversos de su saber lingüístico: «[...] para crear y entender lo que es posible en una lengua, hay que conocer el sistema de la lengua correspondiente; para hablar efectivamente una lengua como los nativos, hay que conocer también la norma o las normas de la lengua misma» (ib., $108 \mathrm{~s}$.).

La gramática contrastiva tal como la concibe Coseriu es una gramática descriptiva (sincrónica) comparada. Además de fines didácticos puede tener un interés lingüístico de mayor alcance al contribuir, tal como espera el autor, a la investigación de los universales lingüísticos (véase ib., 110 s.).

\section{GRAMÁTICA Y SABER LINGÜÍSTICO}

La teoría de la gramática puede concebirse desde perspectivas diferentes. Se puede considerar como algo objetivo, descriptible como entidad independiente, por ejemplo como un sistema, según el enfoque estructuralista. Pero se puede considerar también como capacidad del sujeto hablante, como lo suponen la gramática transformacional o las diversas posiciones dentro de la lingüística cognitiva. Con respecto a la teoría gramatical de Coseriu se puede decir que en primer lugar hace hincapié en la concepción funcional y estructural. Sin embargo, da cuenta también del sujeto hablante, al hacer patente la correlación entre las estructuras y convenciones de la lengua por un lado y la facultad lingüística por el otro.

Como hemos visto, Coseriu distingue entre gramática en cuanto técnica del hablar («gramática objeto») y gramática en cuanto investigación lingüística («metalenguaje»). La tarea de la gramática como disciplina lingüística consiste en la reconstrucción del saber

\footnotetext{
${ }_{10}$ Véase con respecto a estas distinciones Vintila-Radulescu 1969; Albrecht 1988, XXXIX y Aschenberg 2001, 270-273.
}

Odisea, $\mathrm{n}^{\circ} 3,2003$ 
gramatical del hablante. Podemos preguntarnos así pues qué función tiene este tipo de saber dentro de la competencia lingüística en general.

La teoría del saber lingüístico que propone Coseriu obtiene su base y sus líneas en el marco de su teoría general del lenguaje, porque está estrechamente ligada con sus términos centrales. Conforme a los tres niveles del lenguaje (nivel universal, nivel histórico, nivel individual) y sus tipos de actividades lingüísticas correspondientes (hablar en general, lengua concreta, discurso) Coseriu distingue tres tipos del saber lingüístico:

1. el saber elocutivo abarcando, independientemente de las lenguas concretas, el conocimiento de cómo se habla coherentemente;

2. el saber idiomático refiriéndose al conocimiento de las reglas de una lengua concreta;

3. el saber expresivo que tiene que ver con las reglas de la producción de textos en situaciones concretas y con objetivos comunicativos determinados ${ }^{11}$.

Puesto que la gramática se refiere a los procedimientos semántico-gramaticales de las lenguas concretas, el saber gramatical se sitúa en el nivel histórico. Contiene las reglas de una lengua particular sin considerar las circunstancias de su empleo. Forma parte del saber idiomático, pero no lo representa en su totalidad, porque saber hablar una lengua significa también conocer sus inventarios fonológicos y léxicos y sus reglas de combinación. El saber gramatical atañe a la vez a la norma y al sistema de una lengua. El hablante conoce las técnicas idiomáticas tradicionales y sus reglas subyacentes. Esto quiere decir que la actividad lingüística con respecto al saber idiomático no se restringe a la reproducción de lo ya dicho sino que se pone de manifiesto también en la realización de posibilidades expresivas potenciales que proporciona el sistema. Ni la creatividad ni el cambio lingüístico serían pensables sin el supuesto de un saber del hablante abarcando también las regularidades del sistema.

Con respecto a las tres secciones o tres tipos de la gramática (gramática constitucional, funcional y relacional) Coseriu menciona explícitamente que corresponden a lo que sabe el hablante del empleo gramatical de su lengua. El saber gramatical contiene conocimientos de tipo diverso, que en el uso concreto de la lengua no se realizan sucesivamente sino simultáneamente:

1. El hablante conoce los medios de expresión y sus combinaciones que puede o debe emplear para designar un cierto estado de cosas o un cierto contenido de pensamiento (gramática constitucional);

2. Sabe intuitivamente el significado semántico gramatical de las expresiones usadas (gramática funcional);

3. Sabe además cuándo puede elegir entre diversas construcciones gramaticales equivalentes que denominan el mismo estado de cosas (gramática relacional) (véase Coseriu 1987, $145 \mathrm{f}$ ).

Coseriu da un ejemplo francés para ilustrar los diversos aspectos del saber gramatical: mon livre, ma maison. El hablante conoce el adjetivo posesivo y sus combinaciones morfosintácticas. Esto corresponde al saber constitucional. Sabe además que estas expresiones significan relación de dependencia genérica o posesividad; este saber atañe a la

\footnotetext{
${ }^{11}$ Coseriu ha presentado su teoría de la competencia lingüística en diversos escritos: Véase sobre todo Coseriu 1988; 1981, 272 ss.; 1990, 10 ss.
} 
gramática funcional. El hablante conoce también otras posibilidades de expresión refiriéndose a la misma situación, por ejemplo ce livre m'appartient, ce livre est à moi. Conoce así pues la relación entre los diversos paradigmas, conoce sus equivalencias. Este saber forma parte de la gramática relacional (véase ib.).

En el marco de la teoría general de Coseriu de la competencia lingüística el saber gramatical constituye un componente del saber idiomático. Pero, como subraya el mismo Coseriu, el hablar concreto no se basa solamente en la competencia lingüística, exige también otros tipos de saber refiriéndose, a hechos no lingüísticos: contextos objetivos, situaciones, conocimiento general del «mundo» (id. 1981, 275 s.).

\section{CONCLUSIÓN}

La teoría gramatical de Coseriu, más bien un esbozo que una concepción detallada, está fundada por su teoría general del lenguaje. Coseriu desarrolla la idea de un sistema gramatical, cuyas partes son complementos de un todo. La primera sección, la gramática constitucional, representa una morfología general que analiza las relaciones sintagmáticas de los significantes en los diversos niveles de la lengua: unidades mínimas, palabras, grupos de palabras, oraciones y textos. La segunda sección, la gramática funcional, constituye el centro de la gramática; tiene orientación semasiológica y expone los significados de los procedimientos gramaticales al analizar las oposiciones patentes en los paradigmas respectivos de los diferentes niveles de la lengua. La tercera sección es la gramática relacional, de orientación onomasiológica. Estudia las relaciones entre diversos paradigmas al poner de manifiesto unidades semántico gramaticales que designan el mismo estado de cosas o el mismo contenido de pensamiento.

Destaca en los proyectos más concretos de gramática (gramática transfrástica, gramática contrastiva) el esfuerzo por una coherencia conceptual: Coseriu retoma categorías y principios elaborados en la teoría general gramatical para aplicarlos a estos dos tipos de gramática. La gramática transfrástica se refiere al nivel superior de la estructuración de la lengua. Estudia los medios de expresión que traspasan el límite de la oración al emplear categorías de estructuración válidas también en los otros niveles de la lengua como subordinación, superordinación, coordinación, y sustitución. La gramática contrastiva, basada igualmente sobre categorías de la gramática funcional, no es otra cosa que una gramática comparada sincrónica, sirviendo no sólo a fines didácticos sino también al estudio de los universales del lenguaje.

Corresponden a las diversas secciones de la gramática aspectos diversos de la competencia gramatical del hablante. Con respecto al modelo de los diversos niveles del saber lingüístico la competencia gramatical debe atribuirse al saber idiomático, puesto que se refiere a la lengua concreta. Con respecto al hablar concreto, el saber lingüístico representa un solo componente relacionado con otros componentes, es decir con entornos y tipos de saber desarrollados más en detalle en «Determinación y entorno» (1955/56) y en la lingüística textual (cf. $1994^{3}, 124$ ss.).

Odisea, $n^{\circ} 3,2003$ 


\section{BIBLIOGRAFÍA}

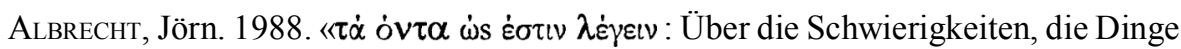
zu sagen wie sind, und andere davon zu überzeugen». Coseriu 1988: XVII-XLV.

Aschenberg, Heidi. 2001. «Typologie als Charakterologie». Martin Haspelmath et al. eds. Language Typology and Language Universals. An International Handbook. Tomo I/ 1: 266-274.

2002. «Eugenio Coseriu als Hochschullehrer». Adolfo Murguía, ed.. Sprache und Welt. Festgabe für Eugenio Coseriu zum 80. Geburtstag. Tubinga: Narr.

Coseriu, Eugenio. 1955/56. «Determinación y entorno. Dos problemas de una lingüística del hablar». Romanistisches Jahrbuch VII. 1955/56: 29-54.

-1968. Einführung in die Transformationelle Grammatik. Vorlesung gehalten im Sommersemester 1968 an der Universität Tubinga. Autorisierte Nachschrift besorgt von Gunter Narr und Rudolf Windisch. Tubinga.

-1976. Das romanische Verbal system. Tubinga: Narr.

1977. Estudios de lingüística románica. Madrid: Gredos.

1981. Lecciones de lingüistica general. Madrid: Gredos..

-1984. Funktionelle Syntax. Vorlesung gehalten im Sommersemester 1983. Nachschrift von Heinrich Weber. Tubinga.

-1987. Formen und Funktionen. Studien zur Grammatik. Tubinga: Niemeyer.

—1987²(1978). Gramática, semántica, universales. Madrid: Gredos.

-1988. Energeia und Ergon. Sprachliche Variation - Sprachgeschichte Sprachtypologie. I. Schriften von Eugenio Coseriu (1965-1987). Eingeleitet und bearbeitet von Jörn Albrecht. Tubinga: Narr.

-1990. Grundzüge der Grammatiktheorie. Nachschrift besorgt von Heinrich Weber. Tubinga.

-19943 (1980). Textlinguistik. Eine Einführung. Herausgegeben und bearbeitet von Jörn Albrecht. Tubinga / Basel: Francke.

GAUGER, Hans-Martin. 1982. «Bedeutung und Bezeichnung». Helmut Stimm/Wolfgang Raible, eds. Zur Semantik des Französischen. Beiträge zum Regensburger Romanistentag. Wiesbaden: Steiner: 25-29 (=ZfSL, Beiheft 9).

KabAteK, Johannes / MurguíA, Adolfo. 1990. «Die Sachen sagen, wie sie sind...». Eugenio Coseriu im Gespräch. Tubinga: Narr.

Lehmann, Christian. 1988. «Zu Eugenio Coserius Sprachtypologie». Jörn Albrecht / Jens LüDTKE / Harald Thun (eds.): Energeia und Ergon. Sprachliche Varietäten Sprachgeschichte - Sprachtypologie, III. Tubinga: Narr: 3-22.

Murguía, Adolfo, ed. Sprache und Welt. Festgabe für Eugenio Coseriu zum 80. Geburtstag. Tubinga: Narr. 
Seppänen, Lauri. 1982. «Bedeutung, Bezeichnung, Sinn. Zur Sprachauffassung Eugenio Coserius». Neuphilologische Mitteilungen 83: 329-338.

SPence, N.C.W.. 1960. «Towards a New Synthesis in Linguistics: The Work of Eugenio Coseriu». Archivum Linguisticum 12,1, 1960: 1-34.

Vintila-RAdulescu, Iona. 1969. «Eugenio Coseriu et la théorie du langage». Revue Roumaine de Linguistique 14: 179-187. 